\title{
A fast algorithm of two-level banded Toeplitz systems of linear equations with application to image restoration
}

\author{
Skander Belhaj ${ }^{1}$ and Marwa Dridi ${ }^{2}$ \\ ${ }^{1}$ Manouba University, ISAMM, Manouba, Tunisia \\ ${ }^{2}$ University of Tunis El Manar, ENIT-LAMSIN, Tunisia \\ Received: 1 July 2016, Accepted: 3 April 2017 \\ Published online: 17 August 2017.
}

Abstract: A fast algorithm is proposed for the solution of $n m \times n m$ block banded Toeplitz systems with banded Toeplitz blocks (BBTBTB). The algorithm consists of using the idea of embedding a BBTBTB matrix in a $m \times(n+k)$ block-triangular Toeplitz matrix. The computational complexity of the proposed algorithm is $O\left(m^{2} n \log n+m^{3} n+k^{3} m^{3}\right)$. The validity of the approach is illustrated by an application to image restoration.

Keywords: Toeplitz matrices, Block-tridiagonal Toeplitz matrices, Block-triangular Toeplitz matrices.

\section{Introduction}

We study the linear system

$$
T x=f
$$

where $T$ is an $N \times N$ block banded Toeplitz matrix with banded Toeplitz blocks (BBTBTB) of the form

$$
T=\left(\begin{array}{cccccccc}
T_{0} & T_{-1} & \ldots & \ldots & T_{-k} & 0 & \ldots & 0 \\
T_{1} & T_{0} & \ddots & \ddots & \ddots & T_{-k} & \ddots & \vdots \\
\vdots & \ddots & \ddots & \ddots & \ddots & \ddots & \ddots & 0 \\
T_{k} & \ddots & \ddots & \ddots & \ddots & \ddots & \ddots & T_{-k} \\
0 & \ddots & \ddots & \ddots & \ddots & \ddots & \ddots & \vdots \\
\vdots & \ddots & \ddots & \ddots & \ddots & \ddots & \ddots & T_{-1} \\
0 & \ldots & 0 & T_{k} & \ldots & \ldots & T_{1} & T_{0}
\end{array}\right)
$$

in which all $T_{j}, j=-k, \ldots, 0, \ldots, k$ are $m$-by- $m$ matrices, $N=n m, x$ is the unknown vector and $f$ is the right hand side. This kind of matrix is encountered, in fields like signal and image processing [8,9]. Very often these matrices are accompanied by a Toeplitz structure of the blocks (See $[6,7,12]$ and reference therein). Among the best known methods, the block-reduction cyclic reduction is the most popular algorithm for solving a BBTBTB system [4]. Another fast method based on the non-standard $Q R$ factorization of the matrix $T$ is given in [15]. A very extensive study for the problem of solving linear system with banded Toeplitz structure can be founded in [10]. In the scalar case, this problem was studied recently via the technique of embedding the matrix $T$ as a triangular Toeplitz matrix [2] and by using a fast 
inversion of a triangular Toeplitz matrix [1].

The main aim of this paper is to propose a method for solving BBTBTB systems based on the idea of embedding block banded Toeplitz matrix with banded Toeplitz blocks (1) in a larger block-triangular Toeplitz matrix [3]. From this point, we shall present some nice properties for a block triangular Toeplitz matrix. Let

$$
L=\left(\begin{array}{cccc}
L_{0} & & & \\
L_{1} & L_{0} & & \\
\vdots & \ddots & \ddots & \\
L_{n-1} & & L_{1} & L_{0}
\end{array}\right)
$$

in which all $L_{j}, j=0, \ldots, n$, are $m$-by- $m$ matrices.

If $m=1$ the inversion of $L$ by forward substitution method [13] can be computed by $O\left(m^{2} n^{2}+m^{3}\right)$ ops. By the use of FFT (Fast Fourier Transformation), a fast exact inversion method based on the divide-and-conquer method [11], requires $O(n \log n)$. If we use the technique of divide-and-conquer to solve block-triangular Toeplitz systems, we need about $O\left(m^{2} n \log n+m^{3} n\right)$ ops and $O\left(m^{2} n\right)$ of the storage. When $m$ is very large, this technique is not better than the block forward substitution method witch costs $O\left(m n^{2}\right)$ ops and $O(m n)$ storage requirement; for more details see [5].

Below a superfast algorithm which computes the inverse of a block lower triangular Toeplitz matrix.

Algorithm 1.1: Block lower triangular Toeplitz matrix inversion

Input: $k$ integer and $m \times m$ block $L_{0}, \ldots, L_{n-1}, n=2^{k}$.

Output: The first block column $v_{n}$ of $L_{n}^{-1}$.

1. Set $v_{1}=L_{0}^{-1}$.

2. For $i=0, \ldots, k-1$

(a). $h=2^{i}$.

(b). Compute the block Toeplitz matrix-vector products $w=S_{h} v_{h}$ and $u=-L\left(v_{h}\right) w$ where $S_{h}$ are $h \times h$

Toeplitz matrices and $L\left(v_{h}\right)$ is the lower triangular Toeplitz matrix whose first column is $v_{h}$ (See [5, p. 36]).

(c). Set

$v_{2 h}=\left[\begin{array}{c}v_{h} \\ u\end{array}\right]$.

So, the inversion of the block triangular Toeplitz system $L_{n}^{-1}$ can be solved in $O\left(m^{2} n \log n+m^{3} n\right)$. In fact, in the Step 2 which appears in both the block Toeplitz matrix-vector products can be computed with the block-DFT (Block Discrete Fourier Transform) in $O\left(m^{2} n \log n+m^{3} n\right)$ (For more details, see [5]).

\section{Remark.[5]}

(i) Let $B_{n}$ and $A_{n}$ be two block lower (upper) triangular Toeplitz matrices. Then $B_{n} A_{n}$ is also a block lower (upper) triangular Toeplitz matrix, and $B_{n} A_{n}=A_{n} B_{n}$.

(ii) If $A_{n}$ is an invertible lower (upper) triangular Toeplitz matrix, then $T^{-1}$ is also a lower (upper) triangular Toeplitz matrix. Therfore, to obtain $T^{-1}$ we only require to compute the entries of its first block-column (block-row).

(iii) If the block Toeplitz matrix $A_{n}$ is not triangular, its inverse is not block Toeplitz.

In this paper, we give a new algorithm for solving BBTBTB systems and motivate our approach by an application to image restoration. The computational complexity in the case one uses fast block-triangular Toeplitz techniques is equal to $O\left(m^{2} n \log n+m^{3} n\right)$ where the blocks are $m$-by- $m$ Toeplitz matrices and $n$ is the order of the BBTBTB matrix. 
The paper is organized as follows: Section 2 gives our main result. In section 3 we illustrate our method with examples given by an implementation of the procedures using MATLAB and we propose a connection with an application in image restoration to show the effectiveness of this algorithm in terms of speed, stability and robustness. Finally, a summary and future research are given in section 4 to complete the paper.

\section{Main algorithm}

Our main idea for solving a BBTBTB is based on the technique of embedding the matrix $T$ as a block triangular Toeplitz matrix. This was firstly proposed by D. Bini, and M. Capavani [3].

Proposition 1. Let $T$ be a BBTBTB as in (1). Then, $T$ can be extended in a block lower banded triangular Toeplitz matrix of the form

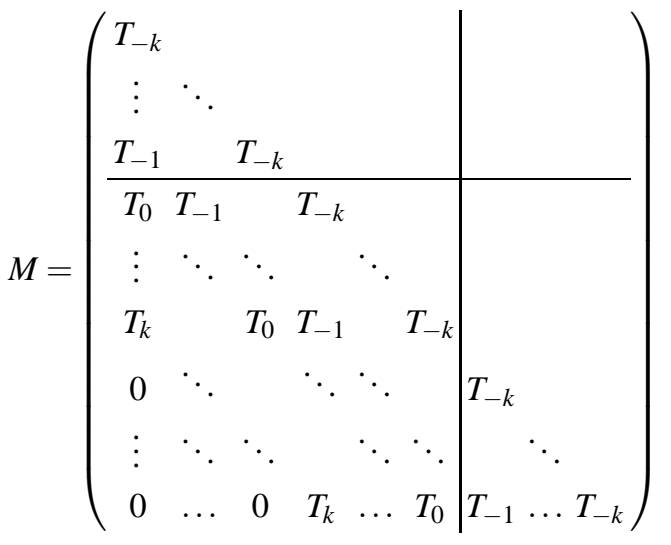

$$
\begin{aligned}
& =\left(\begin{array}{ccc|c}
L & 0 & \ldots & 0 \\
\hline & & \vdots \\
T & & 0 \\
& & & L
\end{array}\right)
\end{aligned}
$$

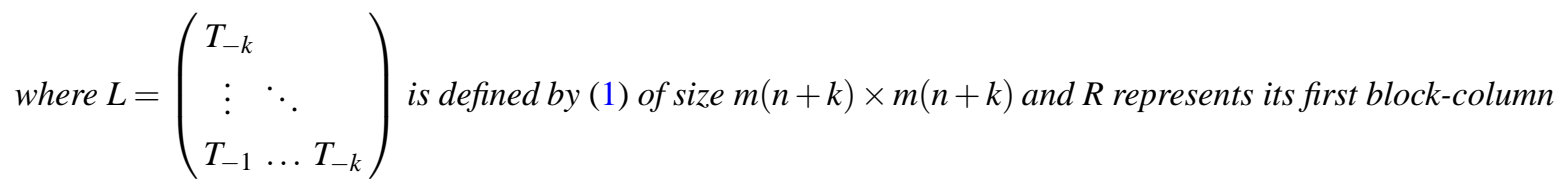

$$
R=\left(T_{-k}, \ldots, T_{-1}\right)
$$

Remark. It is well known that the inverse of a block lower triangular Toeplitz matrix is still a block lower triangular Toeplitz matrix. So, let

$$
V=\left(\begin{array}{ccccc|c}
V_{0} & & & & \\
\hline V_{1} & V_{0} & & & \\
V_{2} & V_{1} & V_{0} & & \\
\vdots & \ddots & V_{1} & V_{0} & & \\
V_{n-1} & & \ddots & \ddots & \ddots & \\
V_{n} & V_{n-1} & \ldots & V_{2} & V_{1} & V_{0}
\end{array}\right)
$$


be the matrix inversion of $M$ defined by its first block-column $\left(V_{0}, V_{1}, \ldots, V_{n}\right)$, where $V_{i}$ is a $m$-by- $m$ matrix not necessary Toeplitz and $i=0, \ldots, n$.

Theorem 1. Let $T$ be a nonsingular block banded Toeplitz matrix with banded Toeplitz blocks (BBTBTB) and M the associate block triangular embedding matrix. Suppose that $M$ is partitioned as follows

$$
M=\left(\begin{array}{ll}
A & B \\
C & D
\end{array}\right)
$$

where $A, B, C$, and $D$ are block matrices of size $n m \times k m, n m \times n m, k m \times k m$ et $\mathrm{km} \times \mathrm{nm}$, respectively. Then, the inverse of $T$, defined by the Schur complement of the block $C$, is given explicitly by

$$
T^{-1}=-A C^{-1} D+B
$$

Proof. We may inductively apply the same proof in [2].

Thus, the algorithm for solving the BBTBTB system is given as follows :

\begin{tabular}{l} 
Algorithm 1: BTT matrix inversion \\
\hline 1. Choose $f$ and compute the inverse of $L$. \\
2. Compute $e_{1}=D f$. \\
3. Solve $C e_{2}=e_{1}$. \\
4. Compute $x_{1}=A e_{2}$. \\
5. Compute $x_{2}=B f$. \\
6. Compute $x=-x_{1}+x_{2}$.
\end{tabular}

Proposition 2. The complexity to solve a TBBTB system (1) is about $O\left(m^{2} n \log n+m^{3} n+k^{3} m^{3}\right)$ ops.

Proof. The inverse of the triangular matrix $M$ requires $O\left(m^{2} n \log n+m^{3} n\right)$. To recover $e_{2}$, we need $O\left(k^{3} m^{3}\right)$ ops where $k$ is the size of the matrix $M . x_{2}$ is provided by $O\left(m^{2} n \log n+m^{3} n\right)$ ops. Thus, the complexity of the system (1) is about $O\left(m^{2} n \log n+m^{3} n+k^{3} m^{3}\right)$ ops.

\section{Numerical tests and application to image restoration}

We have run our numerical tests in MATLAB on the Intel processor E8500 (3.16 GHz). The results given in Tables 1 and 2 show the computational time and the relative error between the computed $\tilde{x}$ by Algorithm 1 and $x$ the exact one (here, the right-hand vector of the system has been chosen so that the system has the solution $\left.x=(1, \ldots, 1)^{T}\right)$.

Example 1. This example is chosen so that $n=2^{12}-1, T_{-1}=T_{1}=\operatorname{circulant}([20-81$ zeros $(1, m-5)-18],-1)(\mathrm{A}$ circulant matrix), $T_{0}=I_{m}$.

\begin{tabular}{l||l|l}
$\mathrm{m}$ & $\|x-\tilde{x}\| /\|x\|$ & Times(s) \\
\hline 5 & $6.37 .10^{-14}$ & 0.025 \\
10 & $4.26 .10^{-14}$ & 0.032 \\
20 & $1.82 .10^{-13}$ & 0.077 \\
30 & $1.45 .10^{-13}$ & 0.150
\end{tabular}


Example 1. This example is chosen so that $n=2^{10}-1, T_{0}=\operatorname{toeplitz}([0.5$, ones $(1, m-1)])$ (A Toeplitz matrix), $T_{1}=$ $T_{-1}=\operatorname{toeplitz}([\operatorname{ones}(1, m / 2)$, zero $(1, m / 2)])$ (A Toeplitz matrix).

\begin{tabular}{l||l|l}
$\mathrm{m}$ & $\|x-\tilde{x}\| /\|x\|$ & Times(s) \\
\hline 10 & $5.64 .10^{-12}$ & 0.126 \\
20 & $5.08 .10^{-12}$ & 0.309 \\
30 & $1.94 .10^{-11}$ & 0.580
\end{tabular}

\subsection{Application to image restoration}

The problem of image restoration has a specific structure which depends on the imposed boundary conditions. In this section, we consider the Dirichlet zero boundary conditions. Let $X=\left(x_{i, j}\right)_{i=1 \ldots, m, j=1 \ldots, n}$ is the original image and $B$ is the blurred image.

Definition 1. The Point-Speared-Function (PSF) array $P$ is the image of a single white pixel, and its dimensions are usually much more than those of $B$ and $X$, if the blurring is local and spatially invariant, then $P$ contains all information about the blurring throughout image.

Definition 2.[16] The zero boundary condition implies a block boundary, so that the pixels outside the borders of the image $X$ are all zeros. That is, we assume that the image $X$ has been extracted from a larger image which looks like

$$
\left[\begin{array}{lll}
0 & 0 & 0 \\
0 & X & 0 \\
0 & 0 & 0
\end{array}\right] .
$$

The blurred image $B$ can be written by a linear system as $b=A x$ where $A$ is a block banded matrix with $m \times m$ banded Toeplitz blocks when the block Toeplitz is derived by proprieties of the Point-Speared-Function (PSF), $b=\operatorname{vec}(B)$ and $x=\operatorname{vec}(X) . A$ is a block $(2 M+1)$-diagonal matrix with $(2 M+1)$-diagonal blocks $[4,14]$. When choosing a PSF as $3 \times 3$ matrix, we get $A$ a BTT matrix.

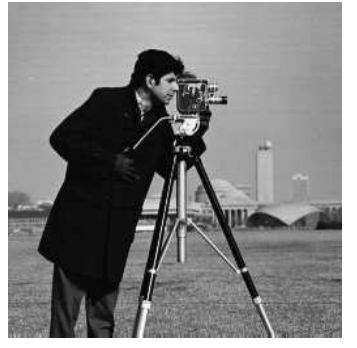

(a) Original image
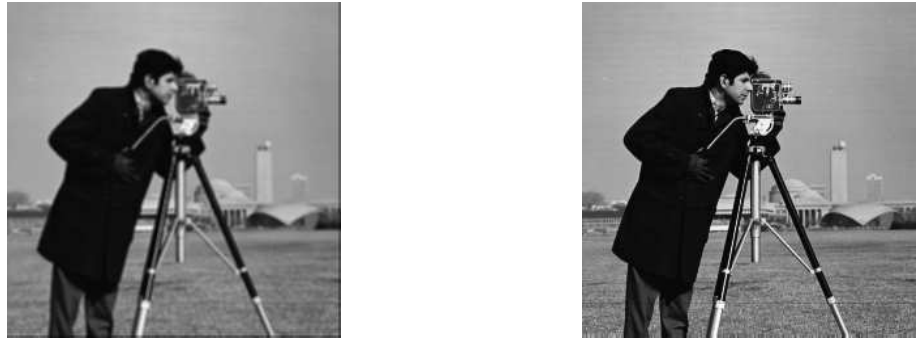

(b) Blurred image (c) Restored image

Fig. 1: Deblurring gray image: Cameraman of size $256 \times 256$. 


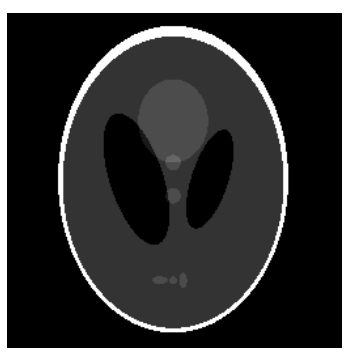

(a) Original image



(b) Blurred image

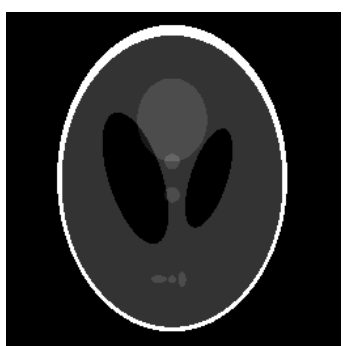

(c) Restored image

Fig. 2: Deblurring gray image: Phantom of size $256 \times 256$.

Algorithm 1 has been implemented and tested for a problem of image restoration of $N \times N$ pixels images, with the PSF defined by the following mask

$$
\mathrm{PSF}=\frac{1}{9}\left[\begin{array}{lll}
1 & 1 & 1 \\
1 & 1 & 1 \\
1 & 1 & 1
\end{array}\right]
$$

which generates a 3-block diagonal block Toeplitz matrix with blocks of size $N$; the off diagonal blocks are tridiagonal Toeplitz matrices with all the nonzero entries equal to $1 / 9$, the diagonal blocks are tridiagonal Toeplitz matrices having $1 / 9$ on the main diagonal and $1 / 9$ on the remaining nonzero diagonals. The matrix obtained in this way is reblocked into a block tridiagonal form, thus obtaining (1). Here, we consider the cameraman in figure 1 with size $256 \times 256$ and the phantom displayed in figure 2 cropped down to size $256 \times 256$.

\section{Concluding remarks}

In this paper a fast algorithm, in $O\left(m^{2} n \log n+m^{3} n+k^{3} m^{3}\right)$ flops, was proposed for the solution of $n m \times n m$ block banded Toeplitz systems with banded Toeplitz blocks (BBTBTB). An application to image restoration was also introduced when the imposed boundary condition is zero. In the future, we tend to focus our attention to solve the problem of image restoration with other boundary conditions such that periodic, reflective (also called Neumann or symmetric) and antireflective.

\section{Competing interests}

The authors declare that they have no competing interests.

\section{Authors' contributions}

All authors have contributed to all parts of the article. All authors read and approved the final manuscript. 


\section{References}

[1] Skander Belhaj and Marwa Dridi. A note on computing the inverse of a triangular Toeplitz matrix. Applied Mathematics and Computation, 236(0):512-523, 2014.

[2] Skander Belhaj, Marwa Dridi, and Ahmed Salam. A fast algorithm for solving banded toeplitz systems. Comput. Math. Appl., 70(12):2958-2967, December 2015.

[3] D. Bini and M. Capovani. Tensor Rank and Border Rank of Band Toeplitz Matrices. SIAM J. Comput., 16(2):252-258, April 1987.

[4] Dario Bini and Beatrice Meini. Solving Block Banded Block Toeplitz Systems with Structured Blocks: New Algorithms and Open Problems. In Michael Griebel, Svetozar Margenov, and Plamen Y. Yalamov, editors, LSSC, volume 73 of Notes on Numerical Fluid Mechanics, pages 15-24. Vieweg, 1999.

[5] Dario A. Bini, Guy Latouche, and Beatrice Meini. Numerical Methods for Structured Markov Chains (Numerical Mathematics and Scientific Computation). Oxford University Press, Inc., New York, NY, USA, 2005.

[6] Dario Andrea Bini and Beatrice Meini. The cyclic reduction algorithm: from Poisson equation to stochastic processes and beyond. Numerical Algorithms, 51(1):23-60, 2009.

[7] B. L. Buzbee, G. H. Golub, and C. W. Nielson. On Direct Methods for Solving Poisson's Equations. SIAM Journal on Numerical Analysis, 7(4):627-656, December 1970.

[8] R. Chan and X. Jin. An Introduction to Iterative Toeplitz Solvers. Society for Industrial and Applied Mathematics, 2007.

[9] Raymond H. Chan and Michael K. Ng. Conjugate gradient methods for toeplitz systems. SIAM Rev., 38(3):427-482, September 1996.

[10] A. Chesnokov and V. M. Barel. A direct method to solve block banded block Toeplitz systems with non-banded Toeplitz blocks. J. Comput. Appl. Math., 234(5):1485-1491, jul 2010.

[11] D. Commenges and M. Monsion. Fast inversion of triangular toeplitz matrices. Automatic Control, IEEE Transactions on, 29(3):250-251, Mar 1984.

[12] Giuseppe Fiorentino and Stefano Serra. Multigrid Methods for Symmetric Positive Definite Block Toeplitz Matrices with Nonnegative Generating Functions. SIAM Journal on Scientific Computing, 17(5):1068-1081, 1996.

[13] Gene H. Golub and Charles F. Van Loan. Matrix Computations. The Johns Hopkins University Press, 3rd edition, 1996.

[14] Per Christian Hansen, James G. Nagy, and Dianne P. O’Leary. Deblurring Images: Matrices, Spectra, and Filtering (Fundamentals of Algorithms 3) (Fundamentals of Algorithms). Society for Industrial and Applied Mathematics, Philadelphia, PA, USA, 2006.

[15] A. N. Malyshev. On solution of large systems of linear equations with block-toeplitz banded matrices. Doklady Mathematics, 87(2):153-155, 2013.

[16] James G. Nagy, Katrina Palmer, and Lisa Perrone. Iterative Methods for Image Deblurring: A Matlab Object-Oriented Approach. Numerical Algorithms, 36(1):73-93, 2004. 